\title{
Molecular Characterization of Salmonella spp. from Cattle and Chicken Farms in Uganda
}

\author{
Takiyah A. Ball, Paula J. Fedorka-Cray*, Joy Horovitz and Siddhartha Thakur
}

North Carolina State University College of Veterinary Medicine, Raleigh, NC, USA

\section{Objective}

Determine the AMR phenotypes and genotypes of Salmonella isolates recovered from cattle and poultry farms in the Wakiso District of Uganda.

\section{Introduction}

Antimicrobial resistance (AMR) is a major concern in developing countries. Uganda is one of many developing countries that are beginning to implement a surveillance program countrywide to monitor AMR within the animal, environmental, and human sectors. Not only is there a concern for AMR, but the emergence of multidrug resistance (MDR) of Salmonella is also becoming a major One Health problem. Few new drugs are being produced. When current treatments fail, new antimicrobials for treatment of these microorganisms are limited (5). In Salmonella, AMR genes are usually found on plasmids that are transferable. Most plasmids that carry resistance are conjugative plasmids, promoting the transfer of DNA from cell to cell (1). Class I Integrons are located on transposable plasmids and are known to transfer AMR through an assortment of gene cassettes (3). Extended-spectrum $\beta$-lactamases (ESBLs) are also known to encode genes located on integrons and transposons (2). ESBLs confer resistance to third generation cephalosporins, a drug of choice for treatment of Salmonella infections. ESBLs are now reported in Enterobacteriaceae all over the world. Examples of common ESBLs include $b l a_{\mathrm{CTX}-\mathrm{M}}, b l a_{\mathrm{OXA}}, b l a_{\mathrm{TEM}}, b l a_{\mathrm{CMY}}$, and $b l a_{\mathrm{SHV}}$ (2). It has been reported that ESBLs evolved from the Kluverya species chromosome by mutation and gene transposition (4).

In our previous study, we phenotypically characterized Salmonella from cattle and poultry farms within the Wakiso District of Uganda. Based on the high prevalence of MDR in the isolates collected we continued investigating at the molecular level. For the Salmonella isolates, we wanted to characterize genotypes by first analyzing the relatedness of the isolates with pulse field gel electrophoresis (PFGE). Next, we wanted to look to see which DNA plasmids were present. We looked at 28 replicon plasmids and the Class 1 Integron, Int1. The Salmonella isolates were also screened for ESBL genes based on their resistance profiles.

\section{Methods}

Fecal and environmental samples from cattle and poultry farms were cultured using standard laboratory methods. AMR profiles were identified among all poultry and cattle Salmonella using the Sensitire ${ }^{\mathrm{TM}}$ system per manufacturer's directions. Fifty-six Salmonella isolates were screened for 28 replicon type plasmids, ESBL genes, and Class I integrons by PCR. The 56 isolates were subjected to PFGE to determine relatedness.

\section{Results}

Salmonella was recovered from 51/379 (13.5\%) and 5/400 (1.3\%) of poultry and cattle samples, respectively. Salmonella Enteritidis 16/51 (31.7\%) and Kentucky 11/51 (21.6\%) were most often recovered on poultry farms. Salmonella was most often resistant to Tetracycline and Sulfisoxazole. All Salmonella Kentucky isolates were resistant to Ciprofloxacin. Five replicon plasmids were identified among all poultry and cattle Salmonella: IncFIIS 18/56 (32.1\%), IncI1 $\alpha$ 12/56 (21.4\%), IncP 8/56 (14.3\%), IncX1 8/56 (14.3\%), and IncX2 1/56
$(1.8 \%)$. The Class I integron, Int1, was positive in one poultry isolate presenting MDR. PFGE cluster analysis of the 56 isolates showed 17 distinctive cluster types and displayed distinct clusters by replicon types IncP, IncX, IncFIIS, and IncI1 $\alpha$. No isolates displayed the ESBL genes that were screened.

\section{Conclusions}

In conclusion, we observed some degree of association between the AMR and plasmids. These plasmids also have an association with the PFGE cluster types and the Salmonella serotypes presented in this study. These Salmonella serotypes may be harboring these particular plasmids which confer resistance to select antimicrobials. Future work with these isolates will include whole genome sequence screening to detect differences between AMR phenotypes and genotypes.

\section{Keywords}

Antimicrobial resistance; Surveillance; Salmonella spp; One Health

\section{Acknowledgments}

We would like to acknowledge funding from the North Carolina State College of Veterinary Medicine and the WHO AGISAR Secretariat. Acknowledgement goes to our collaborators at the Makerere College of Veterinary Medicine in Uganda, Dr. Francis Ejobi, Samuel Maling, David Apollo Munanura, Allan Odeke, Disan Muhangazi, Sarah Tegule, Mark Ogul, Mutumba Paul, Elizabeth Basemera, and Dr. Eddie Wampande in the Central Diagnostic Laboratory and his staff. Acknowledgment also goes to Dr. Megan Jacobs and her staff at the NC State College of Veterinary Medicine Diagnostic Laboratory. We would like to recognize our colleagues Dr. Glenn Tillman, Dr. Mustafa Simmons, and Mary Crews at the USDA in Food Safety and Inspection Service and Dr. Kim Cook, and Jodie Plumblee, in the USDA Bacterial Epidemiology and Antimicrobial Resistance Unit, in Athens Ga.

\section{References}

1. Bennett, P. M. (2008). Plasmid-encoded antibiotic resistance: acquisition and transfer of antibiotic resistance genes in bacteria. Br J Pharmacol, 153 Suppl 1, S347-357. doi:10.1038/sj.bjp.0707607

2. Bradford, P. A. (2001). Extended-spectrum beta-lactamases in the 21st century: characterization, epidemiology, and detection of this important resistance threat. Clin Microbiol Rev, 14(4), 933-951, table of contents. doi:10.1128/cmr.14.4.933-951.2001

3. Fluit, A. C., \& Schmitz, F. J. (2004). Resistance integrons and superintegrons. Clin Microbiol Infect, 10(4), 272-288. doi:10.1111/j.1198743X.2004.00858.x

4. Humeniuk, C., Arlet, G., Gautier, V., Grimont, P., Labia, R., \& Philippon, A. (2002). Beta-lactamases of Kluyvera ascorbata, probable progenitors of some plasmid-encoded CTX-M types. Antimicrob Agents Chemother, 46(9), 3045-3049.

5. Ling, L. L., Schneider, T., Peoples, A. J., Spoering, A. L., Engels, I., Conlon, B. P., Lewis, K. (2015). A new antibiotic kills pathogens without detectable resistance. Nature, 517(7535), 455-459. doi:10.1038/nature14098

*Paula J. Fedorka-Cray

E-mail: paula_cray@ncsu.edu 